\title{
Polyaniline Synthesized with Functionalized Sulfonic Acids for Blends Manufacture
}

\author{
Mara Joelma Raupp Cardoso, Martha Fogliato Santos Lima, Denise Maria Lenz* \\ Programa de Pós-Graduação em Engenharia, Energia, Ambiente e Materiais, \\ Universidade Luterana do Brasil - ULBRA, Avenida Farroupilha, 8001, \\ Prédio 29, sala 203, 92450-900 Canoas - RS, Brazil
}

Received: May 18, 2007; Revised: November 20, 2007

\begin{abstract}
Polyaniline (PAni), an electronic conductive polymer, has poor mechanical properties, such as low tensile, compressive and flexural strength that render PAni a non-ideal material to be processed for practical applications. Desired properties of polyaniline can be enhanced by mixing it with a polymer that has good mechanical properties. In this work, PAni was synthesised using functionalized sulfonic acids like camphorsulfonic acid (CSA) and dodecilbenzene sulfonic acid (DBSA) in order to promote PAni doping and improve its solubility, making possible conductive blends manufacture. The different forms of PAni were characterized by infra-red spectroscopy, thermal analysis, scanning electron microscopy and conductivity measurements. A conductive blend composed of PAni/DBSA and lower density polyethylene (LDPE) was obtained via solubilization method and its thermal, morphological and electrical properties were investigated. Concentrations as low as 5 wt. (\%) of PAni was able to lead to electrical conductivities of PAni/LDPE blends in the range of $10^{-3} \mathrm{~S}_{\mathrm{cm}} \mathrm{cm}^{-1}$, showing great potential to be used in antistatic packing, electromagnetic shielding, anti-corrosion shielding or as a semiconductor.
\end{abstract}

Keywords: polyaniline, lower density polyethylene, conductive blends

\section{Introduction}

Electronic conductive polymers (ECP) have been the subject of study as possible organic metals. Many of these polymers often yield higher conductivity once having undergone the doping process. However, the main disadvantage of ECP is their poor mechanical properties such as strength and processability due to stiffness of their backbone. Thus, the unique combination of electronic and mechanical properties of ECP blends with conventional polymers seems to have great promise for many applications ${ }^{1-3}$. The mixing of polymers has become commercially and technologically more important than the synthesis of homopolymers and copolymers in the last decade. Blending allows creating new materials with specific properties for the desired application at low cost. Since the conducting polyblends are stable and retain the mechanical properties of the host polymer, films, fibers, and coatings can be fabricated by solvent evaporation or by melt-processing for use in anti-static applications, for electromagnetic shielding, microwave absorption devices and/or for transparent conducting films ${ }^{4}$.

In polyaniline (PAni), an electrically conductive polymer, a conjugated double bond structure composed by the benzenoid ring between the quinoid imine and the benzenoid amine structures (Figure 1) form the conjugation $\pi$-bond system ${ }^{5}$. The conjugated double bonds permit easy electron mobility throughout the molecule due to electrons delocalization. Among the three oxidation states of PAni, only doped emeraldine-based (EB) form, being a partially reduced and partially oxidized form, is conductive.

According to MacDiarmid et al. ${ }^{6}$, doping is the process by which polymers that are insulators are exposed to charge transfer agent (dopant). PAni is the only conducting polymer whose properties not only depend on the oxidation state but also on its protonation state/doping level and also on the nature of dopants. The electrical conductivity of undoped PAni is reported to be in the range of $10^{-10}$ to $10^{-7} \mathrm{~S} . \mathrm{cm}^{-1}$. Upon acid doping it attains higher values in the range

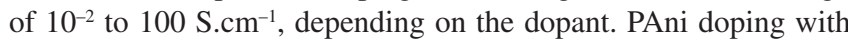
acids improves conductivity levels forming polaron/bipolaron struc- tures that will increase PAni's charge due to the increased electronic delocalization.

Thus, ECP are better semiconductors alternatives to the conventional inorganic such as silicon and bismuth arsenate. The disadvantages of conducting polymers arise from the fact that they are not highly stable compounds even at moderate temperatures $\left(>150{ }^{\circ} \mathrm{C}\right)$ and the most of them exhibit dark colour due to their highly oxidized states, besides their insolubility in the common organic solvents. In order to overcome the above-mentioned drawbacks, it is essential to blend conducting polymers with conventional polymers ${ }^{7}$. Although blending of PAni with other polymers leads to a decrease in its electrical conductivity, it is conceivable that composites or blends with moderate conductivity and good mechanical properties can be obtained by the blending approach ${ }^{8}$.

In this work, conductive blends composed by PAni and low density polyethylene (LDPE) were manufactured via solubilization method. For this purpose, functionalized sulfonic acids in aqueous oxidizing medium were tested as dopants of PAni in order to study their influence in PAni conductivity and solubility. PAni and PAni/ LDPE blend were characterized by scanning electron microscopy, thermal analysis and conductivity measurements. Besides, infra-red spectroscopy was used to identify the conductive bands for PAni samples.

\section{Materials and Methods}

\subsection{Synthesis and characterization of PAni}

The monomer aniline (Merck) was freshly distilled prior to use. After, $0.5 \mathrm{M}$ aniline was chemically polymerized in $1 \mathrm{M} \mathrm{HCl}$ solution using ammonium persulphate $\left(\left(\mathrm{NH}_{4}\right)_{2} \mathrm{~S}_{2} \mathrm{O}_{8}\right)$ as oxidizing agent. The polymerization reaction was kept between 0 and $5{ }^{\circ} \mathrm{C}$ for two hours. The ammonium persulphate concentration was related to aniline concentration by the K parameter, as described in work of Prón et al. ${ }^{9}$ and Lenz et al. ${ }^{10}$. A low value of $\mathrm{K}$ parameter means oxidizing agent 
Conjugated double

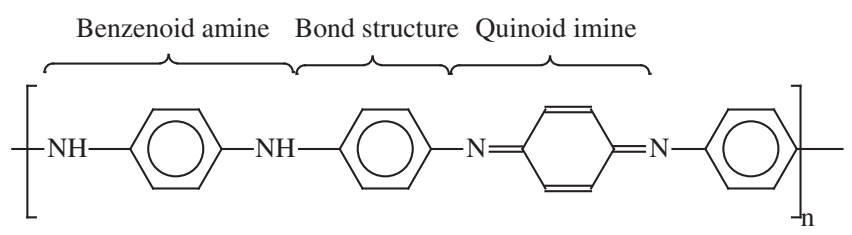

Figure 1. Chemical structure of dedoped PAni (emeraldine-base form).

number of mols in excess with respect to aniline number of mols. PAni powder was then washed and dried. The PAni doped with $\mathrm{HCl}$ acid is called PAni/HCl. Afterwards, $\mathrm{PAni} / \mathrm{HCl}$ powder was neutralized with $0.1 \mathrm{M} \mathrm{NaOH}$ for 24 hours in order to obtain PAni in the dedoped state (insulator state), called PAni/ $\mathrm{NaOH}$. Finally, a doping process was performed in PAni/NaOH using a functionalized sulfonic acid: $1 \mathrm{M}$ dodecil benzenesulfonic acid (DBSA), yielding PAni/DBSA, or $1 \mathrm{M}$ camphorsulfonic acid (CSA), yielding PAni/CSA samples.

Fourier Transform Infrared Spectroscopy (FTIR) was used to identify the specific bands of the different forms of PAni with a FTIR Perkin Elmer spectrophotometer. PAni morphology was investigated using a Philips XL 20 scanning electron microscope and conductivity measurements were carried out at room temperature, following the four probe technique ${ }^{11}$, with a RD2 differential resistometer. Solubility measurements of PAni samples were carried out using organic solvents as toluene and tetrahidrofurane (THF). Thermogravimetric analysis (TGA) and differential scanning calorimetry analysis (DSC) were also used to characterize the different forms of PAni. Thermal studies of PAni are particularly important when one considers the use of elevated temperatures to process PAni and its blends into technologically useful forms. Thermogravimetric analysis (TGA) was performed in a TG2050 thermal analyser in order to find the decomposition temperature of PAni compounds. PAni samples were scanned from 0 to $1000{ }^{\circ} \mathrm{C}$ at a scanning rate of $20{ }^{\circ} \mathrm{C} / \mathrm{min}$ under nitrogen gas purging. DSC was performed using a DSC 2010 differential scanning calorimeter. For DSC experiments, each sample was heated from 30 to $240{ }^{\circ} \mathrm{C}$ at $10{ }^{\circ} \mathrm{C} / \mathrm{min}$.

\subsection{Blends manufacture and characterization}

A PB - 681 low density polyethylene (LDPE) from Braskem (Pólo Petroquímico de Triunfo - RS) was used for blends manufacture. PAni/DBSA and LDPE were dissolved in toluene at $90{ }^{\circ} \mathrm{C}$ under magnetic stirring to form a homogeneous mixture which was cast in glass petri-dish. Blends of LDPE and 1, 2, 5, 10 and 20 wt. (\%) PAni/ DBSA were obtained from the solution by slow solvent evaporation in vacuum stove in order to investigate blend conductivity as function of PAni concentration. Blends were pressed in pellets form in a hydraulic press applying $15 \mathrm{kN} / \mathrm{m}^{2}$ for 10 minutes at room temperature to measure blends conductivity using the four probe method. Further, blends morphology was investigated using scanning electron microscopy. In addition, the thermal properties were evaluated using a TG2050 thermal analyzer equipment from TA Instruments.

\section{Results and Discussion}

\subsection{Synthesis and characterization of PAni}

Concerning the polymerization reaction, higher the K parameter value is, lower is the reaction yield, however PAni conductivity increase. Thus, using a parameter $\mathrm{K}$ value of 0.8 , PAni/HCl samples

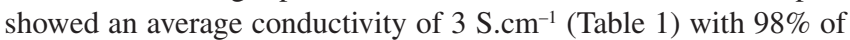
reaction yield while $\mathrm{PAni} / \mathrm{HCl}$ samples synthesized using a $\mathrm{K}$ value
Table 1. Solubility Degree and Conductivity for different forms of PAni.

\begin{tabular}{lccc}
\hline & \multicolumn{2}{c}{ Solubility degree } & \\
\cline { 2 - 3 } & $\begin{array}{c}\text { in Toluene } \\
90^{\circ} \mathrm{C}(\%)\end{array}$ & $\begin{array}{c}\text { in THF } \\
45^{\circ} \mathrm{C}(\%)\end{array}$ & $\begin{array}{c}\text { Conductivity* } \\
\left({\left.\mathrm{S} . \mathrm{cm}^{-1}\right)}^{-1}\right.\end{array}$ \\
\hline PAni/ CSA & 25 & 35 & 0.8 \\
PAni/ DBSA & 30 & 40 & 1.0 \\
PAni/HCl & 9 & 16 & 3.0 \\
PAni/ $\mathrm{NaOH}$ & 20 & 33 & Below $10^{-6}$ \\
\hline
\end{tabular}

*polimerization reactions carried out with $\mathrm{K}$ parameter value $=0.8$ at $0-5{ }^{\circ} \mathrm{C}$ for 2 hours using $\left(\mathrm{NH}_{4}\right)_{2} \mathrm{~S}_{2} \mathrm{O}_{8}$ as oxidant agent.

of 6 showed a conductivity increase to around $15 \mathrm{~S} . \mathrm{cm}^{-1}$. Nevertheless, the reaction yield dropped to only $6 \%$.

In Table 1, the results of PAni solubility experiments can be also found. Although its high conductivity, PAni/HCl has poor solubility in common organic solvents. PAni/DBSA and PAni/CSA showed higher degree of solubility in toluene and THF, making possible blending PAni with other polymers, in agreement with recent works ${ }^{12,13}$. Besides, all forms of PAni were more soluble in THF than in toluene. However, LDPE is weakly soluble in this solvent. Thus, toluene was considered the appropriate solvent for PAni/ LDPE blends manufacture. PAni/DBSA synthesized in operational conditions described in Table 1 (K parameter value equal to 0.8 at $0-5{ }^{\circ} \mathrm{C}$ for 2 hours using $\left(\mathrm{NH}_{4}\right)_{2} \mathrm{~S}_{2} \mathrm{O}_{8}$ as oxidant agent) was also chosen for blends manufacture since PAni/DBSA is the form of PAni that showed the highest solubility degree in both THF and toluene.

In FTIR analysis, there were no significant changes in the bands of PAni doped with dopant agents like $\mathrm{HCl}$, DBSA or CSA. The analysis of the functional groups of $\mathrm{PAni} / \mathrm{HCl}$ and PAni/DBSA by FTIR spectroscopy (Figure 2) showed structural characteristics similar to those reported by Freitas ${ }^{12}$. The main bands are situated around de $820 \mathrm{~cm}^{-1}$ (C-H out-of-plane bending vibration in the 1,4 disubstituted ring), around $1140 \mathrm{~cm}^{-1}$ (vibrational band of the quinoid $\mathrm{N}$ - the electronic band attributed due to charge delocalization on the polymer), in the range of $1240-1300 \mathrm{~cm}^{-1}$ (C-N stretching of the aromatic secondary amines), around $1500 \mathrm{~cm}^{-1}$ (benzenoid stretching) and around $1600 \mathrm{~cm}^{-1}$ (quinoid stretching ).

TGA was used in order to find the decomposition temperature of PAni. This important temperature sets an upper boundary for the temperature at which PAni can be heated for subsequent works. Figure 3 shows the thermograms for the different forms of PAni. PAni/DBSA started to degrade in lower temperature (around $250^{\circ} \mathrm{C}$ ) with respect to other forms of PAni. This fact was also observed by Freitas ${ }^{12}$. For PAni/ $\mathrm{NaOH}$, the TGA curve showed the decomposition beginning at around $500{ }^{\circ} \mathrm{C}$, while for PAni/HCl the decomposition temperature was above $400{ }^{\circ} \mathrm{C}$, after water and $\mathrm{HCl}$ loss.

The acid-doped forms of PAni showed a three step decomposition process, as proposed by previous workers ${ }^{14}$. They suggest that the initial stages of weight loss are due to the volatilization of water molecules and oligomers, as well as unreacted monomer elimination. Then, at higher temperatures the protonic acid component of the polymer is lost and finally at more extreme temperatures the polymer chain break can lead to production of gases such as acetylene and ammonia. $\mathrm{PAni} / \mathrm{HCl}$ was found to be the more thermally stable with respect to PAni/DBSA since the later form showed higher weight losses before the thermal breakdown of the polymer backbone. Emeraldine-base form $(\mathrm{PAni} / \mathrm{NaOH})$ showed after water loss almost a straight line up to its decomposition onset.

DSC curves (Figure 4) for the same forms of PAni showed endothermic peaks: around $130{ }^{\circ} \mathrm{C}$ for $\mathrm{PAni} / \mathrm{NaOH}$ and $\mathrm{PAni} / \mathrm{HCl}$ and around $145^{\circ} \mathrm{C}$ for PAni/DBSA. These endothermic transitions 
are related to the water excess and they are dislocated with respect to TGA experiments since DSC experiments were performed with hermetic pans. The water influence on the polymer crystal structure is poorly studied. Freitas ${ }^{12}$ suggested that water can even be bound to the PAni lattice and Lubentsov et al. ${ }^{15}$ concluded that water influences the crystal structure of PAni and thus changes its conductivity.

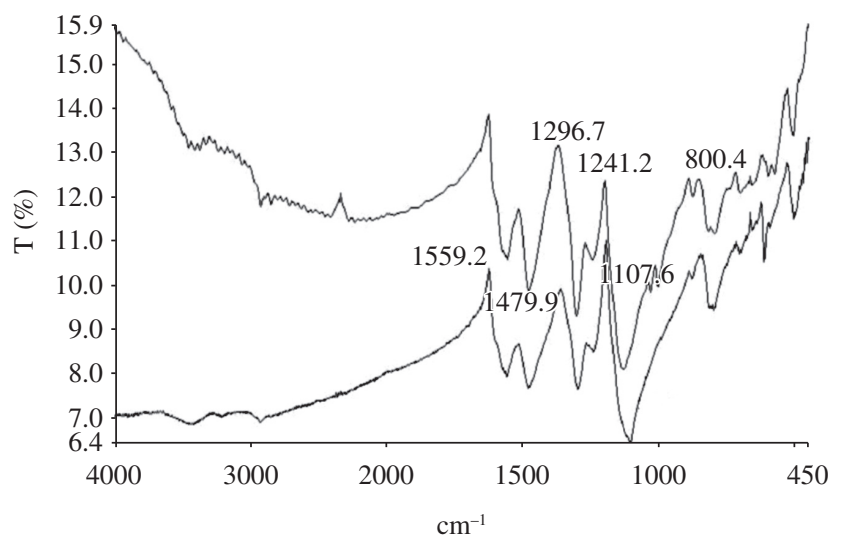

Figure 2. FTIR spectra of PAni/DBSA (bottom line) and PAni/HCl (upper line).

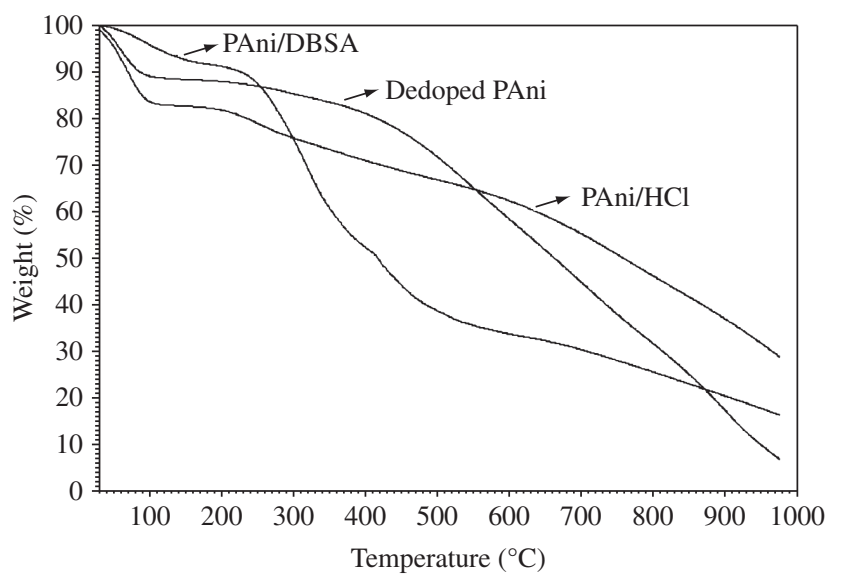

Figure 3. Thermogravimetric analysis for different forms of PAni.

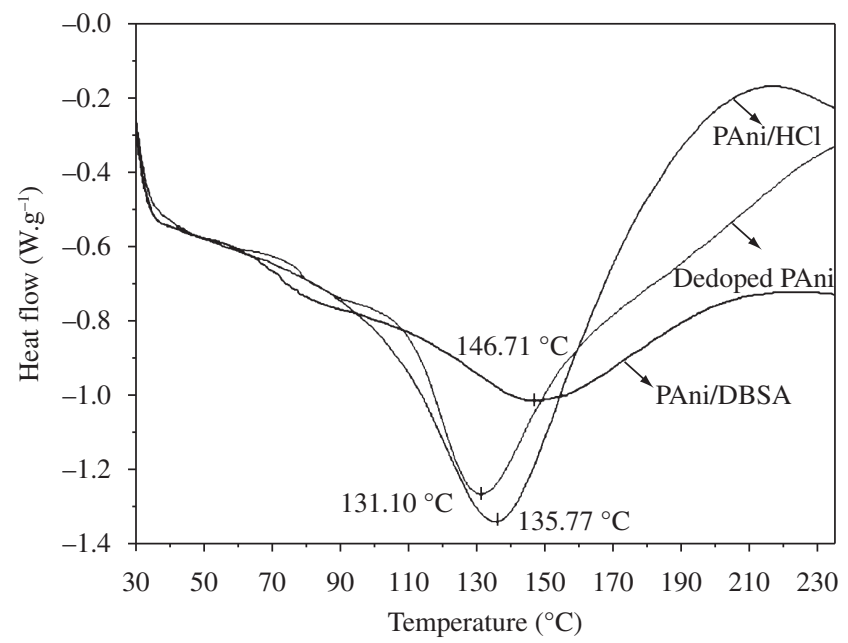

Figure 4. Differential scanning calorimetry analysis for different forms of PAni.
DSC analysis were not performed successfully in PAni's glass transition temperature ( $\mathrm{T}_{\mathrm{g}}$ point) detecting due to the usual difficulties of DSC technique, such as the lack of accuracy for determining this temperature. The $\mathrm{Tg}$ point of pure PAni/DBSA was reported to be around $130{ }^{\circ} \mathrm{C}$, lower than the $\mathrm{Tg}$ of dedoped PAni (over $200{ }^{\circ} \mathrm{C}$ ). This is the result of plasticization effect of the DBSA dopant ${ }^{16}$.

\subsection{Characterization of PAni/LDPE blends}

The conductivity of PAni/LDPE blends increased gradually with the increase of PAni concentration until a stabilization level that indicates the percolation threshold of PAni in LDPE matrix (Figure 5). This percolation threshold was found around $10 \%$ of PAni/DBSA in LDPE matrix. The conductivity of blends composed by LDPE and only 1 and 5 wt. (\%) of PAni/DBSA was in the order of $10^{-6}$ and $10^{-3}{\mathrm{~S} . \mathrm{cm}^{-1}}$, respectively. According to FAEZ et al. ${ }^{17}$, a conductivity range from $10^{-11}$ to $10^{-2} \mathrm{~S}_{\mathrm{cm}} \mathrm{cm}^{-1}$ is considered appropriate to antistatic packaging applications. Blends of LDPE and 5 wt. (\%) PAni were chosen for blends characterization by other techniques.

Figures 6a,b,c show the typical nanosized dot-like aggregates of PAni (white parts) in LDPE matrix also reported by Jianming et al. ${ }^{18}$. In Figure $6 c$ it was possible to observe the fine fibrillar structrure of PAni/DBSA with a higher magnification. According to SEM experiments performed by Zilberman ${ }^{19}$ and Leyva et al. ${ }^{20}$, upper the percolation threshold, the PAni/DBSA structure forms an interconnected matrix through the insulator matrix, allowing the electric conductivity of the mixture in the optimum level. The level of interaction between the PAni and the matrix polymer determines the blend morphology and, thus, its electrical conductivity. Similar solubility parameters of the two polymeric components are necessary for a high level of PAni dispersion within the matrix polymer and, thus, enable the formation of conducting paths at low PAni content. According to Zilberman ${ }^{19}$, PAni can be present in two-structure hierarchy in blends with thermoplastic polymers: a) a primary structure composed of small dispersed polyaniline particles; and b) a shortrange fine fibrillar structure, interconnecting the dispersed particles. Also, these two morphologies were found in this work.

Figure 7 shows thermograms of PAni/LDPE blends. The degradation temperature was around $480{ }^{\circ} \mathrm{C}$, the same as pure LDPE. Thus, the thermal stability of PAni/LDPE blends were the same as the LDPE. Some LDPE samples were processed once in a doublescrew extruder and blended with PAni in the same conditions of the virgin LDPE. No significant change in the degradation temperature was observed by thermogravimetric analysis. DSC analyses in PAni/ LDPE blend (Figure 8) showed only an endothermic peak attributed to its fusion temperature (around $120^{\circ} \mathrm{C}$ ). Again, no marked difference

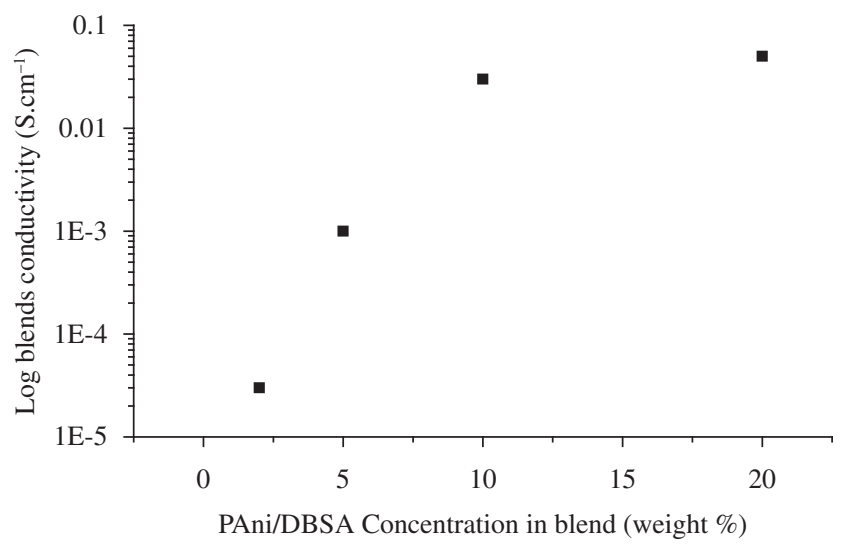

Figure 5. PAni concentration influence on PAni/LDPE blend conductivity. 


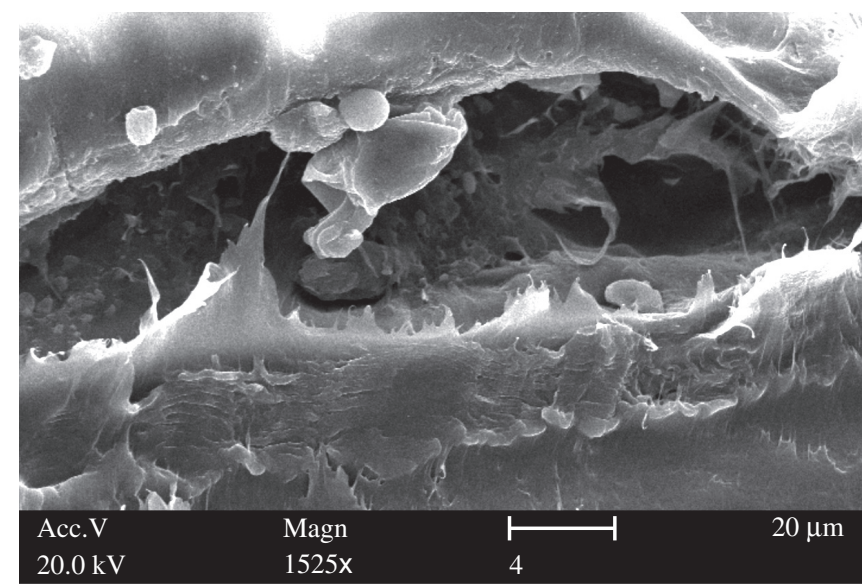

(a)

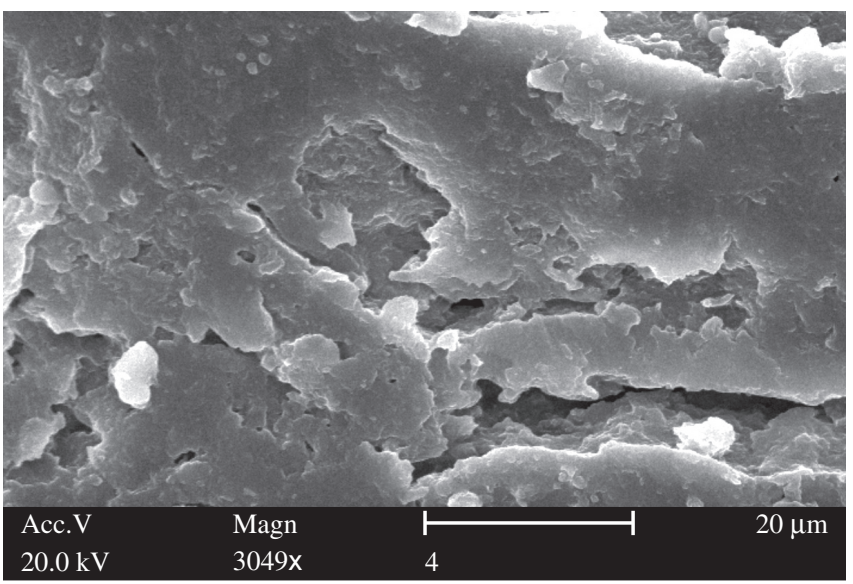

(b)

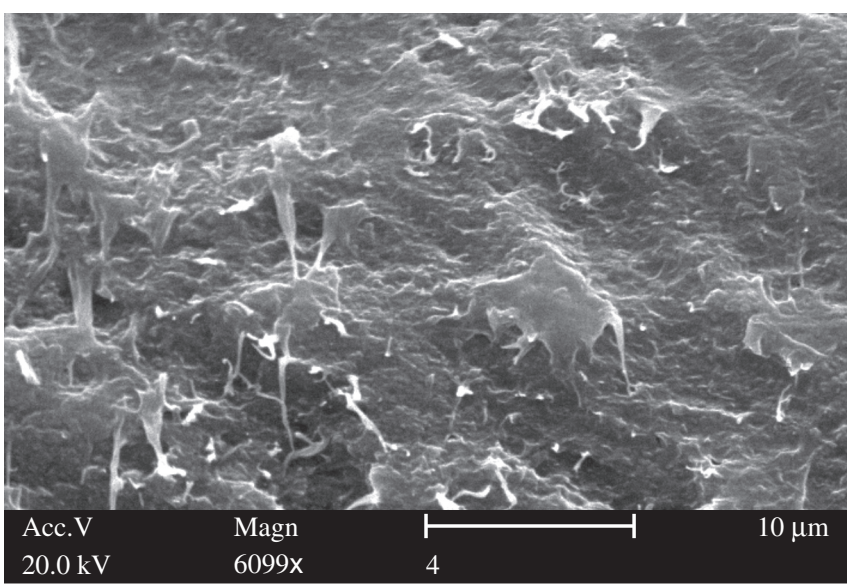

(c)

Figure 6. Scanning electron micrographs of the cross-section of PAni/LDPE blend with 5 wt. (\%) PAni: a) 1500x; b) 3000x; and c) 6000x.

between PAni/LDPE blend and pure LDPE thermal behavior could be detected. Also, blend's fusion temperature was equivalent for either virgin or processed LDPE as blend matrix. The homogeneity of the blends could not be determined accurately through the use of DSC because no Tg value was detected.

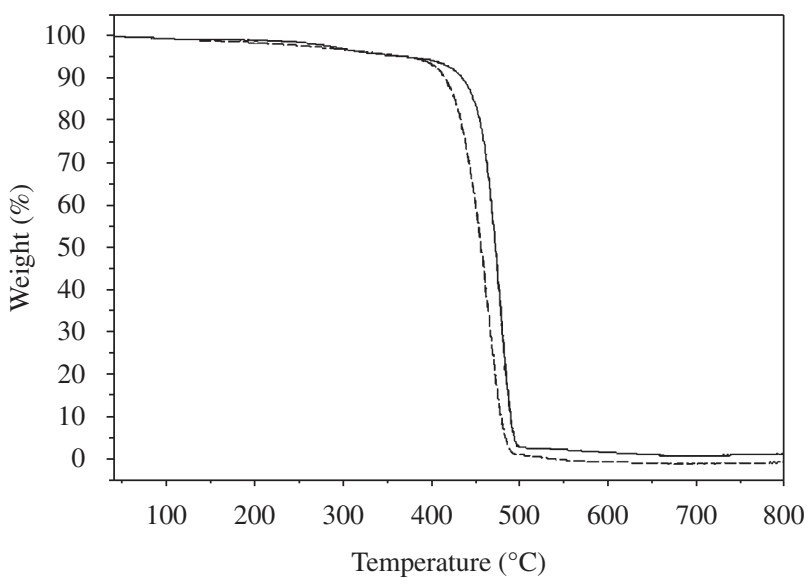

Figure 7. Thermograms of PAni/LDPE blend with 5 wt. (\%) PAni: continuous line - virgin LDPE and dotted line - processed LDPE.

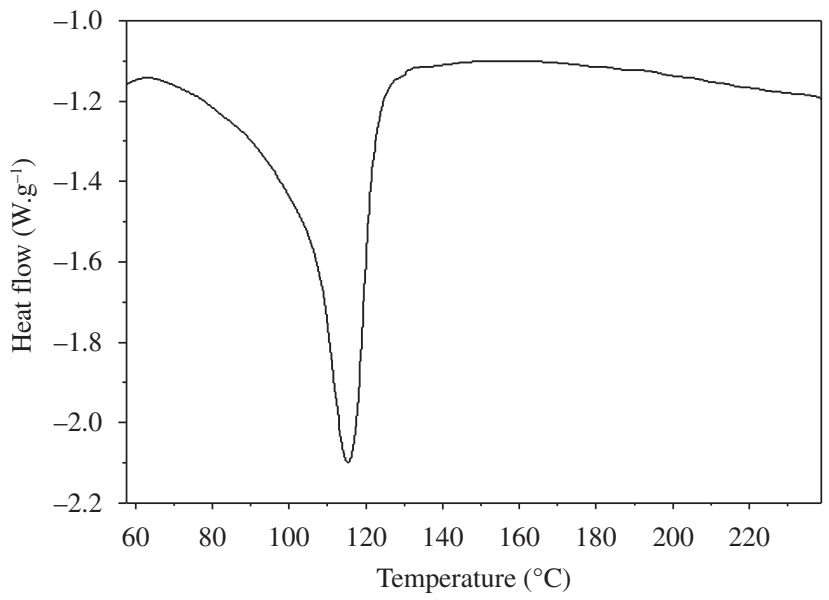

Figure 8. DSC thermogram of PAni/LDPE blend with 5 wt. (\%) PAni.

\section{Conclusion}

Different forms of PAni were synthesised in this work. PAni doped with dodecil benzenesulfonic acid (PAni/DBSA) showed the best combination of solubility and conductivity for blends manufacture via solvent evaporation. However, this form of PAni is less thermal stable, starting its decomposition process at around $250{ }^{\circ} \mathrm{C}$. A blend composed by a mix of 5 wt. (\%) of PAni/DBSA and LDPE showed a degradation temperature of $480^{\circ} \mathrm{C}$ and a conductivity in the order of $10^{-3} \mathrm{~S} . \mathrm{cm}^{-1}$. Thus, the PAni/DBSA has a great potential to be blended with LDPE in order to be used in antistatic packaging. The use of processed LDPE in the blends manufacture presented a new opportunity to increase the aggregated value of the recycled material. The next step of the research is to investigate the thermal properties of the blend by DMTA (dynamic-mechanic-thermal analysis) technique. This information is helpful for processing PAni/LDPE blend in industrial processes like extrusion and injection moulding.

\section{Acknowledgments}

The authors are grateful to Universidade Luterana do Brasil (ULBRA) and FAPERGS for financial support and also to Centro de Microscopia Eletrônica e Microanálise (ULBRA) for SEM micrographs. 


\section{References}

1. Laska J, Żak K, Prón, A. Conducting blends of polyaniline with conventional polymers. Synthetic Metals. 1997; 84(1):117-118.

2. Angelopoulos, M. Conducting polymers in microelectronics. IBM Journal of Research and Development. 2001; 45(1):57-76.

3. Mitzakoff S, De Paoli MA. Blends of polyaniline and Engineering Plastics. European Polymer Journal. 1999; 35(10): 1791-1798.

4. Heeger AJ. Self-assembled Networks of Conducting Polyaniline: A New Class of Conductive Polymer Blends. TRIP. 1997; 3(2):39-46.

5. Epstein AJ. Electrically Conducting Polymers: Science and Technology. Materials Research Society Bulletin. 1997; 16-23.

6. MacDiarmid AG, Chiang JC, Richter AF, Epstein AJ. Polyaniline: A new concept in conducting polymers. Synthetic Metals. 1987; 18(1-3):285-290.

7. De Paoli MA. Handbook of Conductive Organic Molecules and Polymers in: Conductive Blends, v. 2, Nalwa HS, editor. 888 p., New York: John Wiley \& Sons; 1997

8. Barra GMO, Leyva ME, Soares BG, Mattoso LH, Sens M. Electrically conductive, melt-processed polyaniline/EVA blends. Journal of Applied Polymer Science. 2001; 82(1):114-123.

9. Prón A, Genoud F, Menardo C, Nechtschein M. The effect of the oxidation conditions on the chemical polymerisation of polyaniline. Synthetic Metals. 1988; 24(3):193-201.

10. Lenz DM, Schultz MER, Ferreira, CA. Metallization of Polymers by using Conducting Polymers. Journal of Polymer Engineering. 1996/7, 16(4):295-310.
11. Girotto EM, Santos IA. Medidas de Resistividade Elétrica DC em Sólidos: como Efetuá-las Corretamente. Química Nova. 2002; 25(4):639-645.

12. Freitas PS. Sintese da polianilina em escala piloto e seu Processamento. [PhD Thesis] - Universidade Estadual de Campinas, UNICAMP, São Paulo, Brazil, 2000. 156p.

13. Biscaro RS, Botelho EC, Takahashi MFK, Faez R, Rezende MC. Estudo Reológico de Tintas de Poliuretano Contendo PAni-DBSA Aplicadas como Materiais Absorvedores de Microondas (8-12 GHz). Polímeros: Ciência e Tecnologia. 2002; 12(4):318-327.

14. Ansari R, Price WE, Wallace GG. Effect of thermal treatment on electroactivity of polyaniline. Polymer. 1996; 37(6):917-923.

15. Lubentsov B, Timofeeva O, Saratovskikh S, Kirnichnyi V, Pelekh A, Dmitrenko $\mathrm{V}$ et al. Study of conducting polymer interaction with gaseous substances. IV. The water content influence on polyaniline crystal structure and conductivity. Synthetic Metals. 1992; 47(2):187-192.

16. Han MG, Lee YJ, Byun SW, Im SS. Physical properties and thermal transition of polyaniline films. Synthetic Metals. 2001; 124(2-3):337-343

17. Faez R, Rezende MC, Martin IM, De Paoli MA. Microwave properties of EPDM/PAni-DBSA blends. Synthetic Metals. 2001; 119(1-3):435-436.

18. Jianming J, Wei P, Shenglin Y, Guang L. Electrically conductive PANIDBSA/Co-PAN composite fibers prepared by wet spinning. Synthetic Metals, 2005; 149(2-3):181-186.

19. Zilberman M, Titelman GI, Siegmann A, Haba Y, Narkis M. Alperstein D. Conductive blends of thermally dodecylbenzene sulfonic acid-doped polyaniline with thermoplastic polymers. Journal of Applied Polymer Science. 1998; 66(2):243-253.

20. Leyva ME, Barra GMO, Soares BG. Mistura PAni.DBSA/SBS Obtida por Polimerização "In Situ": Propriedades Elétrica, Dielétrica e DinâmicoMecânica. Polímeros. 2002; 12(3):197-205. 